\title{
Metaphorical Processes in English and French within the Source and Target Domain in Cognitive Semantics
}

\author{
Brankica Bojović, PhD, Associate Professor
}

Doi:10.5901/jesr.2014.v4n4p69

Faculty of Philosophy-Nikšić, University of Montenegro

\begin{abstract}
Within cognitive semantics the metaphorical processes, which are very powerful, underlie the mechanism of thought. Semantic changes found in these processes are reflected in the connection between the source and the target domain through the semantic cognitive construction. Note is made of the broadening of the lexical meaning into abstract concepts within English and French cultures which changes the way in which the world is comprehended. The illustrated metaphors are based on their similarity and on cross-domain correlations, which give rise to the perceived similarities between the two domains within the corpus of English and French metaphors.
\end{abstract}

Keywords: metaphors, target domain, source domain, cognitive semantics, language, thought

\section{Introduction}

Cognitive Metaphor Theory has been brought to the centre by a diverse range of scholars within the field of cognitive linguistics. Lakoff and Johnson made it known and widely accepted in their pioneering book Metaphors we live by. This new view of metaphors, i.e. a cognitive linguistic view of the metaphor is developed by Lakoff and Johnson. According to them: a) a metaphor is a property of concepts not words; b) the function of a metaphor is to better understand concepts; c) a metaphor is often not based on similarity; d) metaphors are used in everyday life; e) a metaphor is an inevitable process of human thought and reasoning. This theory (CMT) holds that metaphors are central to thought. CMT's tenets are that: a) metaphors structure thinking; b) metaphors structure knowledge; c) the metaphor is central to abstract language; d) the metaphor is grounded in physical experience; e) the metaphor is ideological. (Deignan 2005:13) Since 1980 up until the present day this topic has been elaborated, because the current interest surpasses the achievements made so far in the field. The stages it passes in psycholinguistics have been developing and it connects language and mind (Chomsky 1968). Descartes' most persuasive arguments of immateriality of the mind includes the belief that thinking is non-physical process. His language philosophy influenced Chomsky's. Metaphor is not only a matter of language but also of thought and reason. It is indeed a topical issue across different disciplines.

\section{Conceptual Mapping Model and Domains}

Ontological mapping across conceptual domains, from the source domain (SD) to the target domain (TD) will be considered here. The mapping in this semantic process is primary because it precisely explains how something is conceptualized and the language is secondary. The source domain consists of a set of literal entities, attributes, processes and relationships, linked semantically and apparently stored together in the mind. These are expressed in language through related words and expressions, which can be seen as being organized into groups resembling those sometimes described as 'lexical fields' by linguists. The target domain tends to be abstract, and takes its structure from the source domain, through the metaphorical link or conceptual metaphor. Also, above all, there are to be observed different definitions of metaphors in the field of cognitive metaphor theory as they are very powerful and natural cognitive processes, which help people understand the complex issues in nature and society and therefore can be perceived as mediators among mind, language and culture. Domain is also described as a semantic structure that functions as the basis for at least one concept. (Croft and Cruse, 2004). The domain TRADE includes the concepts of CUSTOMER, MONEY, SHOP ASSISTANT...

The word metaphor has come to mean a cross-domain mapping in the conceptual system. (Lakoff, 1992).

CMT uses the term 'linguistic metaphor' to refer to the realization of a cross-domain conceptual mapping. In linguistic terms, it defines a metaphor as follows:

A metaphor is a word or expression that is used to talk about an entity or quality other than that referred to by its core, or its most basic meaning. This non-core use expresses a perceived relationship with the core meaning of the word, 
and in many cases between two semantic fields. This general definition can cover a wide range of expressions, including the following:

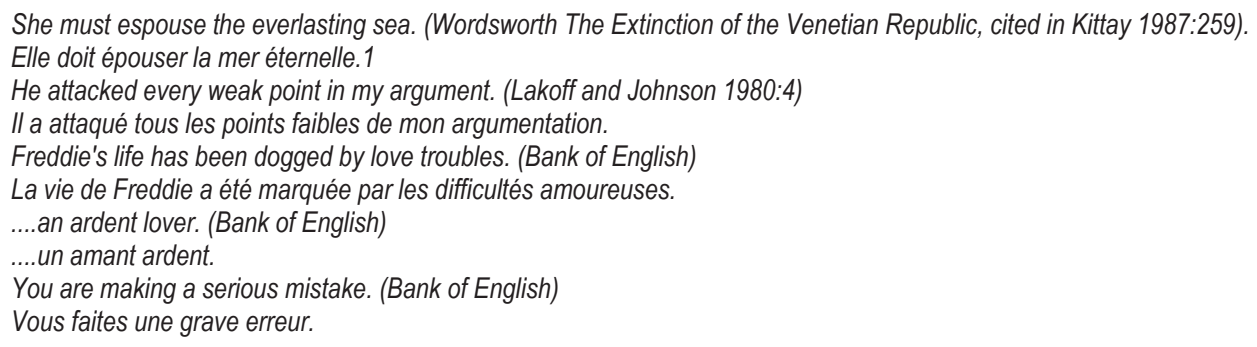

The first of these five expressions realizes A CITY IS A WOMAN, the second, ARGUMENT IS WAR, the third, EMOTIONS ARE ANIMALS, the fourth, EMOTIONS ARE TEMPERATURES, and the fifth a more general mapping of concrete onto abstract. Despite the similarity at the conceptual level, the expressions differ in important ways at the linguistic level. The first expression is an example of innovative linguistic metaphor, in that the word espoused is used unconventionally. The verb is not regularly mapped onto the target domain of cities and its subject is generally human in the source domain. The effect is probably marked for most readers. The second and third expressions, attacked and dogged, are probably unmarked for most language users, because they are regularly used with the meanings they have here. The fourth expression, ardent, is also only used in the sense it has here but unlike dog it has no grammatically related form that is used with a literal meaning in current English. The fifth expression, make, is a type of verb often called "delexical", a label suggesting that it is relatively empty semantically. (Deignan 2005:35)

The wide variety of linguistic metaphors that can realize conceptual mappings is not a problem for conceptual metaphor theorists. On the contrary, it is taken as further proof of the strength of their claims. The existence of the historical metaphors such as ardent is proof that metaphor has shaped the lexicon over centuries, while the exploitation of conceptual metaphors to generate innovative metaphors demonstrates their importance to creative thinking (Lakoff and Turner 1989).

Invariance Hypothesis: Metaphorical mappings preserve the cognitive topology (that is, image-schematic structure) of the source domain. (Lakoff 1990).

Frame is any system of concepts related in such a way that to understand any one of them you have to understand the whole structure in which it fits. (Fillmore, 1982).

A domain may be defined as any knowledge configuration which provides the context for the conceptualization of a semantic unit. (Taylor, 2002:439)

We will explore the role of domains and domain-based knowledge in the semantic structure of words and complex expressions.

Domains vary in complexity from basic conceptions of colour, temperature, space, time, and so on, which cannot reasonably be reduced to other, simpler conceptions, so highly complex knowledge structures, such as the rules of a game, social practices, complex technologies, and typical event scenarios. Most semantic units need to be characterized against more than one domain. The set of domains is called the domain matrix.

Langacker illustrated the notion of domain matrix on the example of the word banana.

\begin{abstract}
Most concepts require specifications in more than one domain for their characterization. The concept [BANANA], for example, includes in its matrix a specification for shape in the spatial (and/or visual) domain; a color configuration involving the coordination of color space with this domain; a location in the domain of taste/smell sensations; as well as numerous specifications pertaining to abstract domains, e.g. the knowledge that bananas are eaten, that they grow in bunches on trees, that they come from tropical areas, and so on. (Langacker,1991:154)
\end{abstract}

He goes on to ask:

Which of these specifications belong to the meaning of the lexical item banana and are therefore included in the grammar of English? Otherwise phrased, which of these specifications are linguistic (or semantic) in nature, and which are extralinguistic (pragmatic)? Which constitute the predicate [BANANA], i.e. the semantic pole of the morpheme

\footnotetext{
${ }^{1}$ All French examples are taken from Professor Anthony Gaudillère (French native speaker)
} 
As the reader might have anticipated, Langacker's answer is that all these specifications belong to the concept [BANANA]. A full characterization of the meaning of the word needs to make reference to these domains and to incorporate them into the semantic value of the expression. Such an approach commits us, inevitably, to an encyclopaedic conception of meaning.

The reason why we need to adopt an encyclopaedic approach is easily stated: we need to appeal to domain-based knowledge in order to account for how words are used. The very fact that we can talk of a bunch of bananas (as compared to the impossibility of talking about *springs of bananas) rests on our knowledge of how bananas grow (and of what bunches look like). If we were to exercise this knowledge from the meaning of banana, we should have to regard bunch of bananas as an arbitrary collocation-or else deny that the collocation is a linguistic fact. Neither option is satisfactory.

An encyclopaedic view certainly does not commit us to the view that each facet of what a person knows is equally central to the concept; some facets are clearly more intrinsic, others may be quite peripheral, while others yet again might be to all intents and purposes irrelevant. The shape, colour, and nutritional value of bananas are probably quite central to the concept and would need to be included in even a summary account of the word's meaning. The fact that people can slip on banana skins is probably rather peripheral, but still capable of being invoked in stereotyped notions of slapstick comedy. Or consider the role of bananas in the economies of small countries in Central America. It is the knowledge that motivates the expression banana republic. It is remarkable the extent to which incidental knowledge about an entity can sometimes have linguistic manifestations. It would be wrong, therefore, to exclude in principle the possibility that even highly contingent aspects of encyclopaedic knowledge could, in certain circumstances, impact on the way a word is used.

There is also a more recent development in cognitive linguistics called Conceptual Blending Theory and the creation of novel metaphors. Blending is when structures from two domains are combined to create new structure. A metaphor is actually a special type of blending. Blending and metaphor create something new.

e. g. This surgeon is a butcher. Ce chirurgien est un boucher.

The above example implies incompetence, though that is not something inherited from either domain.

Lexically speaking a metaphor is defined as an indirect comparison between two or more seemingly unrelated subjects that typically uses "is a" to join the subjects.

A metaphor is sometimes confused with a simile which compares two subjects using "like" or "as".

e.g. He was as sly as a fox. Il a toujours été rusé comme un renard.

While a metaphor would be He was a fox. C'était un renard.

More generally, a metaphor casts a first subject (tenor) as being equal to a second subject (vehicle) in some way. Thus, the first subject can be economically described thanks to the implicit and explicit attributes of the second. A metaphor is sometimes further analyzed in terms of its ground and tension. The ground consists of the similarities between the tenor and the vehicle. The tension consists of the dissimilarities between the tenor and the vehicle. The corresponding terms to tenor and vehicle in Lakoff's terminology are target and source.

In the cognitive linguistic view, metaphor is defined as understanding one conceptual domain in terms of another conceptual domain. Examples of this include when we talk and think about life in terms of journeys, about arguments in terms of war, about love also in terms of journeys, about theories in terms of buildings, about ideas in terms of food, about social organizations in terms of plants, and many others. (Kövecses 2004:22)

Metaphor is a type of analogy and is closely related to other rhetorical figures of speech that achieve their effects via association, comparison or resemblance including allegory, hyperbole, and simile. In simpler terms, a metaphor compares two objects or things without using the words "like" or "as". It is worth mentioning types of metaphors-the following number of types are defined and classified by Lakoff and Johnson $1991^{2}$

\footnotetext{
21 Absolute metaphor - Separated subject and vehicle. An absolute metaphor is one where there is absolutely no connection between the subject and the metaphor. e.g., I am the dog end of every day (It means "To fail to expend the effort needed to do or accomplish something").

2 Dying metaphor - Unfashionable cliché. A dying metaphor is one which has become so over-used. It is considered unfashionable or lacking in eloquence to use it. In effect, it is a cliché. e.g., New era business is a whole different ball game (this means a completely different situation, often one which is difficult or which you know very little about).

${ }^{3}$ Extended metaphor - One subject, many sub-elements. An extended metaphor is one where there is a single main subject to which additional subjects and metaphors are applied. e.g. All the world's a stage and men and women merely players. (This means, life is like a play - we merely go through the stages of our life acting it out.)
} 
Metaphor is noticed in terms of transfer from one cognitive domain or donor to another recipient domain: people tend to draw upon experience in one area of life in order to give fresh insights and understanding to experiences in another, creating new conceptual realities,

e.g. Life is a battle. La vie est un combat.

A wolf in a sheep's clothing. Un loup déguisé en agneau.

Metaphor is the main mechanism through which we comprehend abstract concepts and perform abstract reasoning.

Formally, metaphors are mappings from a source domain to a target domain. Both the source and target domains are structured by schemas and frames.

e.g. I have been feeling quite depressed of late. (happy is up; sad is down)

Metaphor is fundamentally conceptual, not linguistic, in nature. (Lakoff, The Contemporary Theory of Metaphor).

"...metaphorical meaning is not... a special kind of meaning: it is rather the case that metaphor is the result of a special process for arriving at, or construing, a meaning." (Croft and Cruse).

A metaphor is a mapping between conceptual domains. The source domain is the domain supporting the literal meaning of the expression. The target domain is the domain the sentence is actually about. (Croft and Cruse).

Metaphors are mappings across conceptual domains. Mappings are a fixed set of correspondences between entities in a source as well as a target domain. They are grounded in the body and in individual everyday experience and knowledge.

Conceptual metaphor:

e.g. Try to pack more thoughts into fewer words.

Her anger boiled over.

How do you spend your time?

He knows where he is going in life.

Conceptual metaphor involves a relationship between a source domain, the source of the literal meaning of the metaphorical expression, and a target domain, the domain of the experience actually being described by the metaphor:

e.g. Pack thoughts into words. WORDS (target domain) ARE CONTAINERS (source domain)

Anger boiled over. EMOTION/ANGER (target domain) IS HOT FLUID (IN A CONTAINER) (source domain)

Spend time - TIME (target domain) IS MONEY (source domain)

Life is going - LIFE (target domain) IS A JOURNEY (source domain)

The head of the state, the face of the law, a legislative body are examples of body metaphors which are often addressed as metaphors for society.

\footnotetext{
${ }^{4}$ Conceptual metaphors -These are metaphors (or figurative comparisons) in which one idea (or conceptual domain) is understood in terms of another. E.g. variations on the conceptual metaphor - Time is money (it means that time is precious, in a discourse on usury). ${ }^{5}$ Simple metaphor - Single meaning and linkage. A simple metaphor has a single link between the subject and the metaphoric vehicle. The vehicle thus has a single meaning which is transferred directly to the subject. E.g. Cool down! (cool = temperature).

${ }^{6}$ Synecdochic metaphor - This is where one uses a part as metaphor for the whole. A synecdochic metaphor is one where a small part or element of something is used to represent the whole. E.g. Try this nib (meaning to try something new).

${ }^{7}$ Dead metaphor - Offers a comparison that is not symbolic in form, but to physical motion instead. A dead metaphor is simply a comparison that goes unnoticed because the metaphor rests on a comparison that has simply become part of the language. It often involves the use of an idiom. E.g. The committee will hold a meeting (this is a dead metaphor with respect to the word 'hold'; the committee cannot physically grasp the meeting, but the word is being used to equate a physical action with a conceptual one).
} 


\section{Metaphors and Illustrative Examples in English and French}

\begin{tabular}{|l|l|l|}
\hline \multicolumn{1}{|c|}{ Metaphors (in English culture) } & \multicolumn{1}{c|}{ Metaphors (in French culture) } & \multicolumn{1}{c|}{ Transferred meaning } \\
\hline You should plan ahead. & Tu dois prévoir bien à l'avance. & The future is ahead, the past is behind \\
\hline I cannot take my eyes off her. & Je ne peux pas détacher mon regard d'elle. & Seeing is touching/eyes are limbs \\
\hline Her death hit him hard. & Sa mort l'a profondément touché. & Emotional effect is physical contact \\
\hline What are you driving at? & Où veux-tu en venir ? & An argument is a journey \\
\hline She is all heart. & Elle a bon cœur. & $\begin{array}{l}\text { Body parts are symbolic models of stable } \\
\text { meaning in different parts of one's } \\
\text { experience }\end{array}$ \\
\hline $\begin{array}{l}\text { She has been a tower of strength. (She } \\
\text { has been emotionally strong.) } \\
\begin{array}{l}\text { We cemented the agreement with a } \\
\text { toast. (We confirmed our agreement.) }\end{array}\end{array}$ & $\begin{array}{l}\text { Elle a été solide comme un roc. (Elle a été } \\
\text { forte.) } \\
\text { Nous avons scellé notre accord par un } \\
\text { toast. (Nous avons confirmé notre accord.) }\end{array}$ & An argument is a building \\
\hline
\end{tabular}

"Our concepts structure what we perceive... and how we relate to other people. Our conceptual system this plays a central role in defining our everyday realities. If we are right to suggest that our conceptual system is largely metaphorical, then... what we do every day is very much a matter of metaphor." (Lakoff and Johnson, 1980A: 3)

1. Most of our concepts are abstract - concepts like TIME, EMOTIONS, COMMUNICATION, THE MIND, IDEAS...

2. ...Abstract concepts are defined metaphorically in more concrete...terms - concepts like SPACE, MOTION, FOOD, OBJECTS, etc.

3. However, no single, concrete, non-metaphorical concept is ever structured in exactly the right way to completely... define any single abstract concept.

4. As a result... each metaphor defines only certain aspects of an abstract concept. (Lakoff \& Johnson, 1980B)

For example, the concept of an IDEA is defined by a rich and complex cluster of metaphors.

Ideas are people ( $X$ is the father of the idea), ideas are plants (the seeds of his idea were planted in his youth), ideas are products (he produces ideas at an outstanding rate), etc... TIME IS UNDERSTOOD AS MONEY (the flat tyre cost me an hour, I don't have time to give you). ARGUMENT IS WAR (he won the argument, I attacked his position).

Orientational metaphors: GOOD IS UP, BAD IS DOWN (drop dead, at the peak of somebody's health, he fell ill). (Lakoff and Johnson, 1980)

The mapping and conceptual systems are interrelated.

Mapping: a set of ontological correspondences, that characterize epistemic correspondences about one topic onto another.

Mapping - Name: $X$ as $Y$. Love is a Journey.

e.g. LOVE IS A JOURNEY L'AMOUR EST UN VOYAGE

* Lovers correspond to travellers.

*The love relationship corresponds to the vehicle.

*The lovers' common goals correspond to common destinations on the journey.

What constitutes LOVE IS A JOURNEY is not particular words or expression. It is the mapping across conceptual domains.

LOVE = target domain.

TRAVEL = source domain.

LOVE IS A JOURNEY = TARGET DOMAIN IS SOURCE DOMAIN

LE DOMAINE CIBLE EST LE DOMAINE SOURCE

Kövecses allows reverse mappings from TD to SD (p. 25), which seems to be an unnecessary concession. When SD and TD are reversible, it simply means that certain conceptual domains could be SDs sometimes and TDs in other times.

That is, a concept that acts as an SD in one CM can be a TD in another. It should therefore be retained that an SD is always used to understand a TD; this direction is irreversible. What should be allowed for will be that TDs are not always more abstract than SDs. 


\section{Strategies of Translating Metaphors}

For many centuries, metaphor was studied within rhetoric. Evans and Green (2006:303) pointed out that an important idea relates to hiding and highlighting: when a target is structured in terms of a particular source, this highlights certain aspects of the target while simultaneously hiding other aspects. Invoking the metaphor ARGUMENT IS WAR highlights adversarial nature of argument but hides the fact that argument often involves an ordered and organized development of a particular topic (He won the argument, I couldn't defend that point, and so on). In contrast, the metaphor AN ARGUMENT IS A JOURNEY highlights the progressive and organisational aspects of arguments while hiding the confrontational aspects. (We'll proceed in step-by-step fashion. We have covered a lot of ground.) In this way metaphors can perspectivise a concept or conceptual domain.

According to Newmark (1988a:104), "metaphor could be any figurative speech: the transferred sense of a physical word; the personification of an abstraction; the application of a word or collocation to what it does not literally denote, i.e. to describe one thing in terms of another. Note also that metaphor incidentally demonstrates a resemblance, a common semantic area between two or more or less similar things_ the image and the object". He distinguishes six types of metaphor (1988:108): (1) Dead metaphors, whose images are highly unmarked, e.g. at the mouth of the river, (2) Cliché metaphors, which refer to the use of cliché expressions in text, e.g. Long time, no see. (3) Stock or standard metaphors, which he defines as "an established metaphor [...] not deadened by overuse", (4) Adapted metaphors, where the 'fixedness' of a stock metaphor has been adapted or personalised in some way, (5) Recent metaphors, where an anonymous metaphorical neologism has become generally used in the SL. (6) Original metaphors, which are created by the writer or speaker usually to make discourse more interesting and often used to highlight particular points or as reiteration. According to Newmark (1988) there are strategies for translation of metaphors:

1. While dead metaphors are not especially problematical, literal translation is often not possible.

2. In vocative texts, cliché metaphors should be upheld in the TT (ibid.: 107). In informative texts, they should be reduced to their sense or replaced with a more credible stock metaphor.

3. For the translation of stock metaphors, the SL image should be legitimately reproduced in the TL, but the metonyms used may be transferred as long as the substitutes have the same connotations as the SL. However, the SL image is more commonly translated by images that are established to a similar degree. Stock metaphors may also be reduced to sense or literal language.

4. Adapted metaphors should be translated using equivalent adapted metaphors or reduced to sense.

5. Recent metaphors should be translated using componential analysis (ibid.: 114).

6. In vocative texts, original metaphors should be translated literally as they "contain the core of an important writer's message..." (ibid.: 112). If the metaphor is obscure and of little importance to the text, it should be replaced with a descriptive metaphor or reduced to sense. In informative texts, consideration should be given to the number and variety of original metaphors in the text as a whole and a decision be taken between literal translation, reduction to sense or modification of the metaphor.

$\mathrm{He}$ also proposes the translatological strategies for metaphors in the movie industry since there are many difficulties in that field also. These are: 1 . Reproducing the same image in the TL; 2 . Replacing the image in the SL with a standard TL image; 3. Translating metaphor by simile, 4. Translating metaphor by simile + sense; 5 . Converting metaphor to sense; 6 . Deleting; and 7. Combining the same metaphor with the sense.

\section{Summary}

Metaphoricity is the main feature of almost all human languages. According to Lakoff, structurologically considered, metaphors are mappings across conceptual domains. Each mapping is a fixed set of ontological correspondence between entities in a source domain and entities in a target domain. Metaphorical mappings obey the Invariance Principle: The image-schema structure of the source domain is projected onto the target domain in a way that is consistent with the inherent target domain structure. Mappings are not arbitrary, but grounded in the body and in everyday experience and knowledge. There are two types of mappings: conceptual mappings and image mappings. Both obey the Invariance Principle.

In the cognitive linguistic view and figurative creativity analyzed by Kövecses, metaphor is seen as being constituted by a variety of components that interact with each other. The components include: 1 . Experiential basis; 2 . Source domain; 3 . Target domain; 4. Relationship between the source and the target domain; 5 . Metaphoric linguistic expressions; 6. Mappings; 7. Entailments; 8. Blends; 9 . Non-linguistic realization; and 10. Cultural models. A reader can gain insight into choosing any of the strategies mentioned in the paper. 


\section{References}

Banjević Aleksandra, PhD, Associate Professor, University of Montenegro (for consultations on metaphors in the French language). Chomsky N. (1968) Language and Mind, Cambridge University Press.

Croft, W. \& D. A. Cruse (2004) Cognitive Linguistics, Cambridge: Cambridge University Press.

Deignan A. (2005) Metaphor and Corpus Linguistics, Amsterdam, John Benjamins Publishing Company.

Evans V. \& M. Green (2006) Cognitive Linguistics: An Introduction, Cambridge: Cambridge University Press.

Fillmore Ch. (1982) Frame Semantics, in: Linguistics in the Morning Calm. Hanshin Publishing Co., Seoul. 111-137.

Gaudillere Anthony (2014) French examples.

Kittay Eva F. (1987) Metaphor: It's cognitive force and linguistic structure, Oxford, Oxford University Press.

Kövecses Z. (2004) Metaphor: A Practical Introduction, Oxford University Press.

Lakoff G. \& M. Johnson (1980) Metaphors we live by, University of Chicago Press.

Lakoff G. (1990) Cognitive Linguistics: The Invariance Hypothesis: Is abstract reason based on image-schemas? Online Journal (39-74).

Lakoff G. (1992) The Contemporary Theory of Metaphor, Cambridge University Press, online edition.

Lakoff G. et al (1991) The master metaphor list. Technical report, University of California at Berkeley.

Langacker, R. W. (1991) Foundations of Cognitive Grammar, Volume2, Descriptive Application. Stanford: Stanford University Press.

Newmark P. (1988) A textbook of translation, Shanghai Foreign Language Education Press (online edition).

Taylor, J. (2002) Cognitive Grammar. Oxford Textbooks in Linguistics. Oxford: Oxford University Press. 